\title{
BUCHBESPRECHUNGEN
}

\author{
Rudolf VON AlberTini
}

\section{Europäische Kolonialherrschaft 1880-1940}

Zürich, Freiburg i. Br.: Atlantis-Verlag 1976, 527 S.

Der Züricher Historiker, der als Mitherausgeber der Beiträge zur Kolonial- und Úberseegeschichte zahlreiche beachtliche Einzelstudien über die Kolonialgeschichte angeregt und selbst vor geraumer Zeit ein grundlegendes Werk über die Dekolonisation vorgelegt hat, sucht mit dieser Studie eine Lücke in der deutschsprachigen Literatur zu schließen, die zwischen Mommsens Arbeiten über dem Imperialismus und Ansprengers ,,Auflösung der Kolonialreiche“ klafft. Die zeitliche Eingrenzung - insbesondere 1880 als Ausgangsjahr - kann er dabei allerdings nur bedingt einhalten. Die Entwicklung in Indien, Indonesien usw. läßt sich nun einmal schwer ohne Streifzüge ins achzehnte Jahrhundert und früher kaum darstellen. Andererseits wirkt der Abbruch der Darstellung im 2. Weltkrieg, nur gelegentlich mit einigen Hinweisen auf die Zeit danach, oft artifiziell und unbefriedigend. Aber offenbar ist eine solche Gesamtdarstellung einer Universalgeschichte von mehr als zwei Kontinenten eben doch für einen einzelnen schwer zu bewältigen. Viele Kolonien, wie die vorderasiatischen arabischen Protektorate und Mandate, der Sudan, die italienischen Kolonien, Madagaskar, Sierra Leone und Gambia, die Antillen, Guayana u. a. fehlen ganz. Die Darstellung der Entwicklung in den portugiesischen und deutschen Kolonien hat der Autor Albert Wirz überlassen (wobei die Mandatsentwicklung der letzteren gleichfalls ausgespart bleibt). Sicher würde auch einiges dadür sprechen, auch die japanischen (Formosa, Korea, Mandchukuo), amerikanischen (Philippinen, Hawaii, Puerto Rico usw.) und (warum eigentlich nicht?) russischen Kolonien (Zentralasien, Sibirien) nicht aus formalen Gründen einfach auszusparen (,,außereuropäisch") sowie die Politik gegenüber und die Entwicklung in den Halbkolonien zu berücksichtigen. Dies würde allerdings wohl den Rahmen dieser Arbeit sprengen - für die als noch relativ ,,griffige“ $500-$ Seiten-Studie doch auch einiges spricht und eine viel größere verlegerische Konzeption erfordern.

Das Buch ist nach Kolonialregionen gegliedert. Auf Britisch-Indien entfällt allein ein Fünftel des Textes. Die einzelnen Regionalkapitel behandeln meist die Genese und Durchführung der kolonialen Eroberung, das koloniale Herrschafts- und Verwaltungssystem, die Entwicklung der kolonialen Okonomien und schließlich den anti-kolonialen Widerstand und die anti-kolonialen Emanzipationsbewegungen.

Zwei wichtigen Einwänden - die offenbar von Teile des Manuskriptes vorab lesenden Kollegen geäußert wurden - begegnet von Albertini schon in der Einleitung (S. 10): Eine moderne Kolonialgeschichte schreiben, sollte sicherlich von der eigentlichen Aufgabe und Funktion kolonialer Herrschaft, der Ausbeutung der Ressourcen und der Schaffung eines aufnahmebereiten Marktes ausgehen, wobei die Administration in ihrer Funktion für die wirtschaftliche Ausbeutung interpretiert werden müse. Der Umstand der relativen Selbständigkeit der Verwaltungen, die durch ,,bestimmte Sachzwänge“ geleitet wurden - die von Albertini dagegen anführt - muß diesen Ansatz durchaus nicht widersprechen. Tatsächlich zeichnet er das Spannungsverhältnis, das hier sich gelegentlich entwickelte, durchaus auch fruchtbar nach. Die Darstellung der modernen Emanzipationsbewegungen beginnt er meist mit einer Erörterung der Implementierung des modernen Bildungssystems, aus dem die westlich geschulten Eliten als Träger des modernen anti-kolonialen Widerstandes hervorgingen. Damit 
wird die Rolle und Haltung der Volksmassen oft unterbelichtet, ein Einwand, den auch von Albertini selbstkritisch zugesteht.

Mögen diese grundsätzlich kritischen Ưberlegungen durchaus zutreffen, so kann man von Albertini aber durchaus nicht vorwerfen, wie er dies mit einem sarkastischen Seitenhieb auf die durch die Empire sich wenig beeindruckend lassenden Theoretiker (S. 10) selbst tut, eine positivistische Kolonialdarstellung abgeliefert zu haben. Er bemüht sich zwar (durchaus erfolgreich im Rahmen der Grenzen eines solchen Werkes) um eine Gesamtdarstellung der Ereignisse und Fakten. Er diskutiert und interpretiert diese aber durchaus im Lichte der relevanten Theorien der Unterentwicklung und Entwicklung.

Damit hebt sich seine Arbeit wohltuend von den früher üblichen apologetischen Kolonialgeschichten ab, ohne aber der - in jüngster Zeit vorherrschenden - pauschalen und apodiktischen Kritik des Kolonialismus sich anzuschließen. Vielmehr versucht er sehr differenziert und vorsichtig zu argumentieren. Zitatbeispiel aus dem Abschnitt über Algerien: ,, Wohl beanspruchte der Weinbau einen ansehnlichen Teil der Gesamtanbaufläche und hat somit die Expansion des Getreideanbaus eingeschränkt, zumal er ertragreichste Böden in Beschlag nahm, aber der Hektarertrag von Wein ist ein Vielfaches desjenigen von Getreide, und der Weinbau ist bedeutend arbeitsintensiver (etwa 4:1); d. h. selbst bei Saisonarbeit und niedrigen Löhnen hat der Weinbau viel höhere Lohnauszahlungen zur Folge gehabt, als wenn der Boden mit Getreide angebaut worden wäre. . . . Man erkennt hier einmal mehr das Problem oder das Dilemma von Intensivkulturen für den Export, die zwar meist auf Kosten der Nahrungsmittel angebaut werden, pro Flächeneinheit aber einen viel höheren Ertrag abwerfen. Kein Zweifel, daß auch das unabhängige Algerien nach der Vertreibung der Colons den Weinbau pflegt." (S. 221).

Für von Albertini läßt sich so ,,koloniale Wirtschaft" nicht allein mit Begriffen wie ,,Raubwirtschaft und Ausbeutung“ erfassen. ,,Mise en valeur und wirtschaftliche Entwicklung haben stattgef unden, obschon vom ,exportorientierten Wachstum' vor allem die Metropolen profitiert haben und die Entwicklung einseitig blieb. Die Kolonialadministrationen haben insbesondere mit dem Aufbau eines Kommunikationssystems (Post, Telegraph, Eisenbahnen, Häfen und Straßen) Entwicklungsarbeit geleistet, die nicht unterschätzt werden darf, auch wenn diese primär der Offnung des Hinterlandes und damit dem Außenhandel diente." (S. 402).

Obwohl das Kolonialsystem so Teilmodernisierungen bewirkt hat - die man allerdings durchaus unterschiedlich bewerten kann - erkennt auch von Albertini an, daß die Kolonialmächte im Grunde sozial-konservativ eingestellt und um die Aufrechterhaltung der bestehenden Gesellschaftsstrukturen besorgt waren (S. 389). Damit wurden sicherlich die Optio nen für eine eigenständige gesellschaftliche und ökonomische Umwälzung erheblich eingeschränkt. Von Albertini hat aber sicherlich nicht Unrecht, wenn er darauf hinweist, daß daraus nicht gefolgert werden könne, daß es ohne Kolonialherrschaft zu sozialen Revolutionen gekommen wäre (ibidem).

Auch sollte man nicht den Tschad und Indien in diesen Fragen gemeinsam diskutieren. Indien wird ja immer als Beispiel genannt, wie ein Land mit einer blühenden Manufaktur durch die koloniale Eroberung von einem relativ hoch entwickelten Niveau zu einem Rohstoffexporteur zurückentwickelt wurde. Abgesehen davon, daß dies in vielen anderen Ländern in der Form gar nicht möglich war (weil eben die Manufakturen noch nicht so entwickelt waren), entstand auch in Indien noch während der Kolonialzeit, schon ab Ende des 19. Jahrhunderts eine bodenständige Industrie. Diese blieb allerdings auf wenige Sektoren beschränkt (so gab es u. a. Baumwoll-, Textil-, Jute-, Zement- und auch eine Stahlindustrie, kaum jedoch Produktionsmittel- und Chemieindustrien) (S. 58 ff.). Sie vermochte auch nur 
wenige ,fforward and backward linkages“ zu entwickeln. Der koloniale Staat war eben hier nicht bereit, die notwendige Rolle eines Geburtshelfers - durch Zoll-, Kredit- und Fiskalpolitik und sein government spending - zu spielen. In den fehlenden produktiven forward-and backward-linkages zwischen den modernen Export- und Industriebereichen und den zurückgebliebenen ,,traditionellen“ Sektoren - ohne deren ,,symbiotische Verbindung“ zu verleugnen, wie sie in der modernen Entwicklungsforschung unter dem Begriff der strukturellen Heterogenität analysiert wird - sieht von Albertini das Hauptcharakteristikum der kolonialen Situation (S. 394-397). Die Ausbeutungs- und Kapitalabflußthese hält er, etwa in Form des terms-of-trade-Argumentes, für empirisch nicht zwingend erwiesen, in Gestalt der ,,drain-of-wealth“-These zwar für gegeben, aber doch für weithin überschätzt (S. 392-393). Mag von Albertini auch gelegentlich übervorsichtig in seinen Beurteilungen sein (so daß auch der Rezensent ihm nicht immer zu folgen vermag), so ist sein Buch doch ein wichtiger Beitrag zur aktuellen Debatte über das Verhältnis von exogenen bzw. endogenen Faktoren als Determinanten der Unterentwicklung und Entwicklung. Seine Arbeit setzt einen Kontrapunkt zu der im letzten Jahrzehnt modisch gewordenen Überbetonung der negativen Effekte des ,,imperialistischen“ Weltmarktes (in kolonialer und nach-kolonialer Zeit) auf die Entwicklungsperspektiven der Dritten Welt. Diese Phase war sicherlich notwendig und auch verständlich als Reaktion auf die zuvor weithin dominierende kolonial- und weltmarktapologetische Literatur. Sie droht aber inzwischen wissenschaftlich in ihrer Eindimensionalität festzufahren und zudem zunehmend als politisch-ideologisches Instrument mißbraucht zu werden. Es ist der Verdienst von Albertinis, für einen Teilbereich sich hiervon abgesetzt zu haben, ohne gleichzeitig in die alte Kolonialapologetik und den Diskussionsstand von vor zwei, drei Jahrzehnten zurückzufallen.

Rolf Hanisch

\section{GeRHARd LeibHolz (Herausg.)}

\section{Jahrbuch des öffentlichen Rechts der Gegenwart}

Neue Folge, Bd. 27 (1978), J. C. B. Mohr (Paul Siebeck), Tübingen

Die hier anzuzeigende Ausgabe des Jahrbuchs des öffentlichen Rechts ${ }^{1}$ enthält neben neun Beiträgen zu Themata aus europäischen Verfassungsrechten (darunter eine monographische Analyse Boris Meissners zur Bundesverfassung der Sowjetunion von 1977 mit Textabdruck in deutscher Ubersetzung) sowie zu den politischen Parteien Israels vier Arbeiten, die asiatische Staaten betreffen, ferner eine zur Republik Südafrika und den beiden ersten sog. Homelands.

Die Verfassung der Volksrepublik China vom 5. März 1978 wird von Oskar Weggel detailliert beschrieben und mit ihren Vorgängerinnen verglichen. Die Entwicklung des Verfassungsrechts der Republik China schildert Y. S. Weng, Rechtsprofessor in Taipeh. Beide Aufsätze werden ergänzt durch den Abdruck der Verfassungstexte in deutscher bzw. englischer Ubersetzung. Die an der Weimarer Verfassung orientierte Verfassung der Republik China vom 1. Januar 1947 ist auf das gesamte chinesische Staatsgebiet zugeschnitten. Wegen der auf Taiwan beschränkten tatsächlichen Gewalt der Republik ist sie seit 1948 durch sog. Vorläufige Bestimmungen in vielerlei Hinsicht suspendiert worden (,, für die Zeit der Rebellion“, wie Weng in Anlehnung an die Vorläufigen Bestimmungen formuliert). Die zahlreichen Änderungen dieser Bestimmungen schildert der Autor ausführlich und macht so deutlich, wie ein Provisorium zur Entstehung tiefgreifender, auf Dauer angelegter Strukturände-

$1 \mathrm{Zu}$ Inhalt und Eigenart dieses Periodikums sowie den Bänden 23 bis 26 s. meine Besprechung in VRU 12 (1979), S. 165 bis 168. 